Notfall Rettungsmed 2023 · 26:101-107 https://doi.org/10.1007/s10049-021-00930-9 Angenommen: 15. Juli 2021 Online publiziert: 18. August 2021

○ Der/die Autor(en) 2021

\section{Emotionen im Rettungsdienst}

\author{
Emotionale Reaktivität, Empathie und Strategien im \\ Vergleich zu Studierenden
}

\author{
Juliane Völker(D) - Mira E. F. Flohr-Devaud \\ Fachbereich I - Psychologie, Universität Trier, Trier, Deutschland
}

\title{
Zusammenfassung
}

Hintergrund: Rettungskräfte müssen im Notfalleinsatz häufig mit emotional belastenden Situationen und ihren eigenen Reaktionen auf menschliches Leid umgehen. Wir untersuchten Rettungskräfte hinsichtlich ihrer Empathie und emotionalen Reaktivität sowie Strategien zur Emotionsregulation und Bewältigung schwieriger Situationen und stellten Zusammenhänge zum chronischen Stresserleben her. Zum Vergleich diente eine studentische Vergleichsstichprobe, welche keine medizinischen Berufserfahrungen vorwies.

Methode: Stress, Empathie, Emotionsregulation und Bewältigungsstrategien wurden mittels einer Fragebogenbatterie erfasst. Emotionale Reaktivität wurde experimentell mit normiertem Bildmaterial zur Erzeugung von Ekel, Trauer, Angst und Freude untersucht, welches auf einer mehrstufigen Skala von angenehm bis unangenehm beurteilt wurde.

Ergebnisse: Rettungskräfte $(n=161)$ erlebten weniger Stress, waren weniger empathisch und machten seltener oder ähnlichen Gebrauch von Strategien zur Emotionsregulation und Bewältigung wie Studierende $(n=56)$. Sie empfanden zudem ekel- und trauerauslösende Bilder weniger unangenehm. Mehr Reaktivität auf Ekelbilder, mehr Empathie, Emotionsunterdrückung und vermeidende Bewältigungsstrategien waren mit mehr Stress assoziiert.

Konklusion: Eine verminderte, jedoch nicht niedrige Empathie könnte im Einsatz hilfreich zur emotionalen Abschirmung sein, während nach dem Einsatz eine aktive Auseinandersetzung mit eigenen Emotionen und erlebten kritischen Situationen adaptiv zum Schutz vor Stress erscheint.

\section{Schlüsselwörter}

Notfallrettung · Chronischer Stress · Emotionsregulation · Bewältigungsstrategien · Prävention

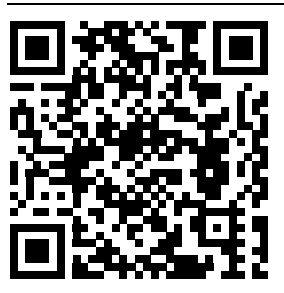

QR-Code scannen \& Beitrag online lesen
Rettungskräfte sind vielfältigen organisationalen, umweltlichen und operatorischen Belastungen ausgesetzt. Dazu zählen Gefahrensituationen, Zeitdruck, medizinische Verantwortung und Kommunikation mit Teammitgliedern, Krankenhauspersonal oder Angehörigen von Betroffenen [4, 6, 15]. Die Bedeutsamkeit organisatorischer und kollegialer Beanspruchungen für gesundheitliche Risiken wie Burn-out ist gut belegt $[6,13,26]$. Doch auch die psychoemotionalen Aspekte des Berufsbilds werden zunehmend berücksichtigt. Im Angesicht von Verletzung, Leid und Tod können Gefühle eigener Inkompetenz, überwältigendes Mitgefühl, gar stellvertretende Traumatisierung auftreten [2, 3, 11, 12, 21, 24, 25]. Aus überdauernder Beanspruchung entstehen Stress, Müdigkeit wider das Mitgefühl und emotionale Zermürbung $[6,21]$ sowie ein Ruf nach Vermittlung von Kompetenzen im Umgang mit emotionalen Belastungen [5]. Unser Ziel war die Darstellung der Empathie von Rettungskräften, ihrer Strategien zur Emotionsregulation und Bewältigung von schwierigen Situationen sowie ihrer Reaktivität auf emotionales Bildmaterial. Zudem untersuchten wir deren Rollen für das chronische Stresserleben und stellten die Befunde einer studentischen Vergleichsgruppe gegenüber. 


\section{Empathie, emotionale Reaktivität} und Emotionsregulation im Einsatz

Affektive Empathie, d.h. Mitgefühl und Unbehagen im Angesicht menschlichen Leids [22], ist unerlässlich für soziale Anteilnahme. Empathie kann in medizinischen Professionen dazu beitragen, die Kommunikation mit Patienten zu verbessern, erlebte Sinnhaftigkeit der Arbeit zu steigern und Burn-out vorzubeugen [18, 28]. Empathie ist jedoch ein zweischneidiges Schwert, denn sie kann auch die Vulnerabilität des Personals steigern. Eine emotionale Verbindung zum Patienten (z. B. bei biografischer Ähnlichkeit, persönlicher Bekanntheit von Opfern oder bei Kindern) erhöht das Risiko, selbst Symptome traumatischen Stresses wie intrusive Bilder, Schlafstörungen oder Erregbarkeit zu entwickeln [3, 24]. Dieses Phänomen ist als sekundäre Traumatisierung bekannt, indem starkes Mitgefühl und Identifizierung mit Opfern einen Transfer der traumatischen, emotionalen Belastung auf den Helfenden verursachen können [25]. So regulieren erfahrene Mediziner Mitgefühl im Anblick fremder Schmerzen bereits vorbewusst und schätzen diese in Folge als weniger intensiv für das Gegenüber ein als eine Vergleichsgruppe ohne medizinische Praxiserfahrungen [7]. Rettungskräfte verzeichnen sogar bereits in der Ausbildung eine niedrigere Empathie als z.B. Pflegepersonal [28]. Wie gut sich Rettungskräfte trotz menschlichen Verständnisses von den Gefühlen der anderen abgrenzen können und somit Kontrolle bewahren, scheint für die Wirkung von Empathie ausschlaggebend zu sein $[3,5]$. Sie können sich z.B. durch volle Fokussierung auf technische, medizinische Aspekte ihrer Arbeit emotional distanzieren und Kontrollgefühle herstellen [3] oder wertfrei wahrnehmen [5].

Im Rettungseinsatz werden Emotionen gelegentlich als ärger-, scham- und schuldbesetzt erlebt $[4,14,21]$. Eine allgemein adaptive Strategie, um aufkommende Emotionen zu regulieren, ist eine Neubewertung der Situation, z.B. als bewältigbar oder indikativ für bestimmte Vorgehen. Jedoch kann auch eine Unterdrückung des emotionalen Erlebens gerade im turbulenten Geschehen dazu beitragen, spätere Intrusionen negativer Erinnerungen an das Ereignis zu reduzieren [8]. Führende Ret- tungskräfte setzen sowohl Neubewertung als auch Unterdrückung stärker ein als weniger erfahrene Einsatzkräfte - sie scheinen durch ihre Erfahrung imstande, ihre Emotionen durch Erkennen und Kontrolle kompetent zu regulieren [4]. Ähnlich berichten Mitmansgruber und Kollegen [21] von erfahrenen Rettungskräften, die die eigenen Emotionen abwerten und verachten, was sie anscheinend vor den direkten, belastenden Erfahrungen kritischer Ereignisse schützt. Beck und Kollegen betonen jedoch auch, dass eine chronisch vermeidende Haltung gegenüber dem Erfahren eigener Emotionen dazu führen könnte, dass man den Umgang mit Gefühl "verlernt" oder "abstumpft", und dies könnte sich auf andere, positive Empfindungen übertragen [4].

\section{Bewältigung nach dem Einsatz}

Kritische Ereignisse verschwinden in der Regel nicht aus dem Gedächtnis, können aber auf verschiedene Arten aufgearbeitet werden. Sehr häufig werden Gedanken und Gefühle für sich selbst behalten oder auf etwas Schöneres wie die eigene Familie oder den baldigen Feierabend abgewen$\operatorname{det}$ [12].Jedoch haben sich vertrauensvolle Gespräche im Team oder mit Nahestehenden als besonders wirkungsvoll erwiesen, um mit emotionalen Belastungen umzugehen $[9,14]$. Das Aufsuchen von Unterstützung, aktives Problemlösen und Akzeptanz erweisen sich oft als hilfreich, während wiederum vermeidende und selbstbeschuldigende Haltungen mit intensiverem Stresserleben zusammenhängen $[2$, 16].

\section{Ziel}

Wir untersuchten Ausprägungen und Zusammenhänge zwischen affektiver Empathie, Emotionsregulation, diversen Bewältigungsstrategien und dem chronischen Stresserleben als Risikofaktor für Burn-out und sekundäre Traumatisierung bei Rettungskräften. Um einem vermeintlichen Abstumpfen $[6,21]$ nachzugehen, interessierten wir uns zudem für die Reaktivität auf emotionales Bildmaterial, welches normativ Ekel, Trauer, Angst oder Freude auslöst (d.h., wie unangenehm es beurteilt wird). Wir überprüften, ob es Unter- schiede zu einer Vergleichsgruppe gibt, welche nicht regelmäßig mit emotional anspruchsvollen Situationen konfrontiert ist.

\section{Methodik}

\section{Stichprobe}

Aus einer rheinland-pfälzischen Rettungsdienst $\mathrm{gGmbH}$ nahmen 161 Personen (97 männlich, $M_{\text {Alter }}=31,2$ Jahre, $S D=10,74)$ mit einer durchschnittlichen Beschäftigungsdauer von 10,57 Jahren $(S D=9,40)$ unentgeltlich teil. Tätigkeitsschwerpunkte lagen in der Notfallrettung $(n=128)$ und dem Krankentransport $(n=33)$. Die meisten Personen absolvierten pro Monat bis zu 15 Dienste à zwölf Stunden $(n=75)$, weitere 67 übernahmen 16 bis 20 Dienste und 19 Personen über 20 Dienste. Als Vergleichsgruppe wurden 56 Universitätsstudierende (21 Männer, $M_{\text {Alter }}=22,64$ Jahre, $\left.S D=3,09\right)$ ohne Erfahrungen in medizinischer Praxis rekrutiert. Post hoc ergibt sich mit $N=217$ eine Teststärke von 0,996 für das experimentelle 4(Bilder: Ekel, Trauer, Angst, Freude) $\times 2$ (Gruppe: Rettungsdienst, Studierende)-Design [10].

\section{Material}

\section{Fragebögen}

Subjektives Stresserleben in den vergangenen drei Monaten wurde mit dem Trierer Inventar zum chronischen Stress ermittelt (zwölf Items, Cronbachs a $=0,89$; Beispielitem betrifft „Befürchtung, meine Aufgaben nicht erfüllen zu können“; [27]). Mitgefühl (vier Items, $a=0,75$; „Mich berühren Dinge sehr, auch wenn ich sie nur beobachte") und Unbehagen (vier Items, $a=0,81$; „Ich fühle mich hilflos, wenn ich inmitten einer sehr emotionsgeladenen Situation bin") als Komponenten von Empathie wurden mit dem Saarbrücker Persönlichkeitsfragebogen erfasst [22]. Der habituelle Einsatz von Neubewertung (sechs Items, $a=0,80$; "Wenn ich mehr positive Gefühle empfinden möchte, versuche ich, über die Situation anders zu denken") und Unterdrückung (vier Items, $a=0,78$; „Ich behalte meine Gefühle für mich") zur Emotionsregulation wurde mit dem Emotion Regulation Questionnaire 
Tab. 1 Mittelwerte (Standardabweichung) der Bewertung des Bildmaterials

\begin{tabular}{|l|l|l|l|l|}
\hline \multirow{2}{*}{ Bildkategorie } & \multicolumn{4}{l}{ Emotions-Rating } \\
\cline { 2 - 5 } & Ekel & Trauer & Angst & Freude \\
\hline Ekel & $6,07(0,99)$ & $2,20(1,05)$ & $2,65(0,64)$ & $1,09(0,10)$ \\
\hline Trauer & $2,29(0,63)$ & $6,09(0,75)$ & $3,00(0,61)$ & $1,13(0,12)$ \\
\hline Angst & $2,74(0,95)$ & $1,84(1,04)$ & $5,49(0,66)$ & $1,25(0,23)$ \\
\hline Freude & $1,26(0,16)$ & $1,19(0,22)$ & $1,27(0,23)$ & $6,66(0,34)$ \\
\hline
\end{tabular}

Anmerkungen. Bewertet durch $N=29$ in einer Vorstudie. Pro Kategorie $n=7$ Bilder. Skala von 1 (äuBerst wenig) bis 9 (äußerst viel)

Tab. 2 Mittelwerte (M) und Standardabweichungen (SD) von Rettungskräften und Studierenden im Vergleich

\begin{tabular}{|c|c|c|c|c|c|c|c|}
\hline \multirow[t]{2}{*}{ Skala } & \multicolumn{2}{|c|}{$\begin{array}{l}\text { Rettungskräfte } \\
(n=161)\end{array}$} & \multicolumn{2}{|c|}{$\begin{array}{l}\text { Studierende } \\
(n=56)\end{array}$} & \multirow[t]{2}{*}{$F(1,215)$} & \multirow[t]{2}{*}{$p$} & \multirow[t]{2}{*}{$\eta_{p}^{2}$} \\
\hline & $M$ & $S D$ & $M$ & $S D$ & & & \\
\hline Stress $^{\mathrm{a}}$ & 2,50 & 0,72 & 3,06 & 0,67 & 25,86 & $<0,001^{* * *}$ & 0,11 \\
\hline \multicolumn{8}{|l|}{ Empathie $^{\mathrm{b}}$} \\
\hline Mitgefühl & 3,28 & 0,68 & 3,88 & 0,59 & 34,44 & $<0,001^{* * *}$ & 0,14 \\
\hline Unbehagen & 2,07 & 0,60 & 2,91 & 0,84 & 66,43 & $<0,001^{* * *}$ & 0,24 \\
\hline \multicolumn{8}{|l|}{ Emotionsregulation $^{\mathrm{c}}$} \\
\hline Neubewertung & 4,33 & 0,98 & 4,51 & 0,96 & 1,51 & 0,220 & 0,01 \\
\hline Unterdrückung & 3,83 & 1,30 & 3,65 & 1,14 & 0,83 & 0,362 & 0,00 \\
\hline \multicolumn{8}{|c|}{ Bewältigungsstrategien $^{\mathrm{d}}$} \\
\hline Akzeptanz & 2,80 & 0,73 & 2,54 & 0,73 & 4,98 & $0,027^{*}$ & 0,02 \\
\hline Aktive Bewältigung & 2,75 & 0,76 & 2,81 & 0,57 & 0,27 & 0,602 & 0,00 \\
\hline Emo. Unterstützung & 2,62 & 0,84 & 2,99 & 0,79 & 8,31 & $0,004^{* *}$ & 0,04 \\
\hline Pos. Umdeutung & 2,59 & 0,74 & 2,61 & 0,60 & 0,02 & 0,899 & 0,00 \\
\hline Ablenkung & 2,53 & 0,68 & 2,62 & 0,67 & 0,70 & 0,404 & 0,00 \\
\hline Instr. Unterstützung & 2,38 & 0,83 & 2,90 & 0,79 & 16,71 & $<0,001^{* * *}$ & 0,07 \\
\hline Humor & 2,06 & 0,79 & 2,10 & 0,84 & 0,08 & 0,773 & 0,00 \\
\hline Selbstbeschuldigung & 2,03 & 0,75 & 2,45 & 0,85 & 11,73 & $0,001^{* *}$ & 0,05 \\
\hline Emo. ausleben & 1,98 & 0,67 & 2,31 & 0,72 & 9,82 & $0,002^{* *}$ & 0,04 \\
\hline Verleugnung & 1,46 & 0,58 & 1,96 & 0,75 & 25,83 & $<0,001^{* * *}$ & 0,11 \\
\hline Religion & 1,40 & 0,72 & 1,41 & 0,56 & 0,02 & 0,901 & 0,00 \\
\hline Alkohol \& Drogen & 1,36 & 0,68 & 1,45 & 0,71 & 0,66 & 0,417 & 0,00 \\
\hline \multicolumn{8}{|c|}{ 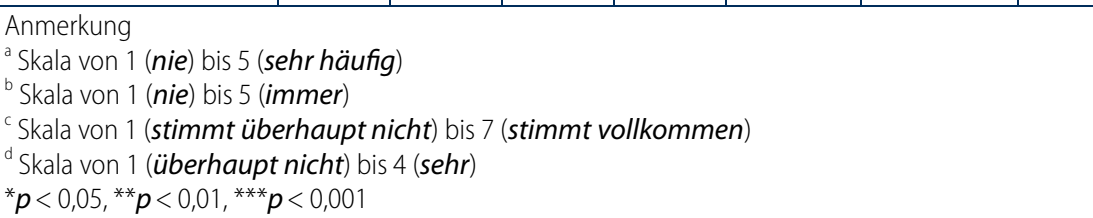 } \\
\hline
\end{tabular}

[1] operationalisiert. Mittels Brief COPE (Kurzversion des Coping Orientation to Problems Experienced Inventars [17]) wurden 14 Strategien zur Bewältigung schwieriger Situationen erhoben, darunter z.B. Akzeptanz („Ich habe gelernt, damit zu leben") und Humor als Indikatoren von positivem Fokus; aktive Bewältigung (,Ich habe aktiv gehandelt, um die Situation zu verbessern") und Planung als aktive Strategien, und Selbstbeschuldigung („Ich habe mich selbst kritisiert und mir
Vorwürfe gemacht") und Verleugnung als vermeidende Strategien (jeweils zwei Items, Spearman-Brown-Koeffizient von 0,63 bis 0,93). Die Skalen für Planung und Rückzug erwiesen sich als unreliabel $(<0,50)$ und wurden nicht analysiert.

\section{Bilder}

Wir verwendeten normierte Bilder aus dem International Affective Picture System [19, 20], einem standardisierten Bilderset zur experimentellen Erzeugung von Emotionen. In einer Vorstudie wählten wir je sieben Bilder aus, die reliabel entweder Ekel, Trauer, Angst oder Freude kommunizieren (- Tab. 1). Ekelbilder zeigten menschliche Verletzungen, aus ethischen Gründen wurden drei extreme Bilder aus alternativen Onlinequellen ersetzt. Trauerbilder stellten Krankheit oder Tod dar. Angstbilder beinhalteten bedrohliche Menschen oder Tiere. Freudebilder zeigten lachende Menschen in positiven Situationen.

\section{Ablauf}

Die gesamte Erhebung wurde online durchgeführt [23]. Teilnehmerlnnen wurden instruiert, jedes Bild einzeln entsprechend ihres spontanen Gefühls auf einer Skala von 1 (äußerst angenehm) bis 9 (äußerst unangenehm) zu bewerten. Die Bilder wurden ebenso wie die sich anschließenden Fragebögen in zufälliger Reihenfolge dargeboten. Die Erhebung dauerte ca. 30 min.

Ethik

DieStudie wurde unter Beachtung der ethischen Richtlinien der WMA durchgeführt [29]. Teilnehmerlnnen wurden vorab über die Inhalte der Studie aufgeklärt und auf die Präsentation von möglicherweise verstörenden Bildern hingewiesen. Sie wurden weiterhin über Anonymität, Freiwilligkeit und ihr Recht informiert, jederzeit ohne Angabe von Gründen zu pausieren oder abzubrechen. Alle Teilnehmerlnnen stimmten der Aufzeichnung und Auswertung ihrer Daten zu.

\section{Ergebnisse}

Die Daten wurden mit SPSS Statistics Version 27 (IBM Corp, Armonk, NY, USA) ausgewertet. Stress, Empathie, Emotionsregulation und Bewältigungsstrategien von Rettungskräften und Studierenden sind in - Tab. 2 gegenübergestellt. Rettungskräfte berichteten weniger Stress und weniger Empathie als Studierende. Emotionsregulation durch Neubewertung oder Unterdrückung sowie sieben der Bewältigungsstrategien wurden von beiden Gruppen gleichsam verwendet, während fünf Strategien (emotionale und instrumentelle Un- 


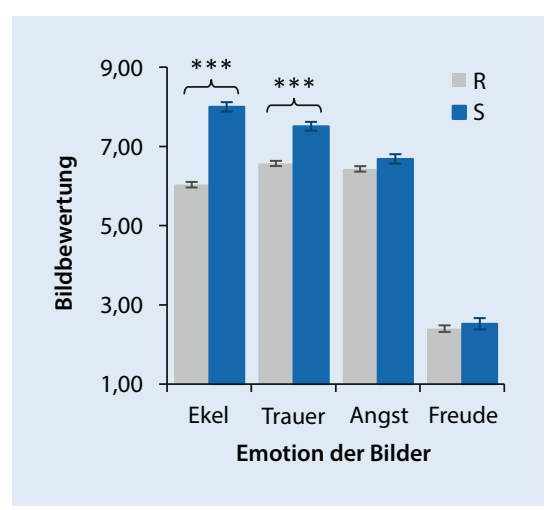

Abb. $1 \Delta$ Mittlere Bewertung der Bilder durch Rettungskräfte (R) und Studierende (S). Fehlerbalken zeigen Standardfehler. Skala von 1 (äuBerst angenehm) bis 9 (äußerst unangenehm). ${ }^{* * *} p<0,001$

terstützung, Selbstbeschuldigung, Ausleben von Emotionen, und Verleugnung) bei Rettungskräften weniger als bei Studierenden zum Einsatz kamen. Einzig Akzeptanz wurde von Rettungskräften stärker angewandt als von Studierenden.

\section{Emotionale Reaktivität}

Als konsistent unangenehm wurden Bilder der Kategorien Trauer $(M=6,81, S D=0,93$, $a=0,80)$, Ekel $(M=6,54, S D=1,24, a=0,90)$ und Angst $(M=6,50, S D=0,90, a=0,73)$ erachtet, während Freude als angenehm empfunden wurde $(M=2,43, S D=1,06$, $a=0,88$ ). Unterschiede zwischen Rettungskräften und Studierenden wurden mit vier einfaktoriellen Varianzanalysen untersucht (• Abb. 1), Konfidenzintervalle wurden wegen multiplen Testens auf $98,75 \%$ angepasst. Rettungskräfte bewerteten Ekelbilder als weniger unangenehm $(M=6,03, S D=0,80)$ als Studierende $(M=8,00, S D=1,10), F(1,215)=203,71$, $p<0,001, \eta_{p}^{2}=0,49$. Auch Trauerbilder wurden von Rettungskräften weniger unangenehm bewertet $(M=6,57, S D=0,79)$ als von Studierenden $(M=7,51, S D=0,94)$, $F(1,215)=52,91, \quad p<0,001, \quad \eta_{p}^{2}=0,20$. Angstbilder wurden von Rettungskräften ähnlich unangenehm erachtet $(M=6,43$, $S D=0,88$ ) wievon Studierenden $(M=6,68$, $S D=0,92), F=3,31, p=0,070$. Bilder mit freudigem Inhalt wurden sowohl von Rettungskräften $(M=2,40, S D=1,10)$ als auch Studierenden $(M=2,52, S D=0,97)$ gleichermaßen angenehm bewertet, $F<1$, $p=0,458$.

\section{Korrelate von Stresserleben}

Signifikante Korrelate von chronischem Stress bei Rettungskräften und Studierenden sind in $\boldsymbol{0}$ Tab. 3 dargestellt. In beiden Gruppen hingen mehr empathisches Unbehagen, mehr Selbstbeschuldigung und weniger aktive Bewältigung mit größerem Stresserleben zusammen. Für Rettungskräfte bedeuteten überdies eine stärkere Reaktivität auf ekelauslösende Bilder, mehr Mitgefühl, stärkere Unterdrückung sowie mehr Einsatz von Ablenkung, Verleugnung und Konsum von Alkohol \& Drogen mehr Stress. Anders als bei Studierenden war Akzeptanz bei Rettungskräften nicht mit weniger Stress assoziiert.

\section{Diskussion}

Kritische, emotionale Ereignisse gehören zum Alltag des Rettungsdiensts. Sie geben dem Berufsbild ihren Sinn und Reiz [28], stellen aber auch große Ansprüche an das Personal. Werden persönliche Ressourcen und Kontrollempfinden ausgereizt, können starke Emotionen die Funktionsfähigkeit beeinträchtigen und überdauernd zu Stress, Burn-out oder sogar sekundärer Traumatisierung führen [3, 12, 24, 25]. In dieser Studie wurden Rettungskräfte und Studierende hinsichtlich der Ausprägungen und Zusammenhänge von chronischem Stress, Empathie, Emotionsregulation und Bewältigungsstrategien sowie der Reaktivität auf ekel-, trauer-, angst- und freudeinduzierende Bilder gegenübergestellt.

\section{Reaktivität, Empathie und Emotionsregulation}

Rettungskräfte fielen konform mit früheren Studien durch weniger Empathie auf $[24,28]$ und empfanden Ekel- und Trauerbilder als weniger unangenehm als Studierende. In der Notfallrettung sind Situationen, die Ekel und Trauer auslösen können, alläglich. Dass Rettungskräfte derartiges Bildmaterial als weniger unangenehm werteten als ihre Vergleichsgruppe, könnte auf eine "harte Schale" aufgrund der chronischen Konfrontation zurückzuführen sein, die jedoch nicht auf Angst oder gar Freude zutrifft. Dass sich also eine chronisch vermeidende Haltung ge- genüber dem eigenen Erleben auch auf andere Emotionen übertragen könnte [4], konnte hier nicht gefunden werden. Dass Rettungskräfte weniger auf Bilder menschlicher Verletzungen reagierten, steht zudem mit weniger erlebtem Stress in Verbindung.

Auch eine geringere Empathie war mit niedrigerem Stresserleben assoziiert. Eine niedrigere Empathie könnte als Schutzmechanismus gegen emotionale Belastung durch Mitgefühl und Unbehagen im Angesicht fremden Leids interpretiert werden [3]. Dennoch bleibt zu betonen, dass Empathie keineswegs niedrig ausfiel. Ein gewisses Maß an emotionaler Verbindung zu Notleidenden scheint immer bestehen zu bleiben, was innerhalb medizinischer Berufe ein sinngebender und motivierender Aspekt ist, bei dem es trotz Mitgefühl mit Anderen auf die Abgrenzung zu den eigenen Emotionen ankommt [3, 18].

Zur Emotionsregulation setzten Rettungskräfte sowohl die Strategie Neubewertung als auch Unterdrückung ähnlich intensiv wie Studierende ein. Die konkreten Werte decken sich mit vorherigen Studien [4]. Bei Rettungskräften war Unterdrückung eigener Emotionen mit chronischem Stress assoziiert. Dass Rettungskräfte vermeiden, sich mit den eigenen Emotionen auseinanderzusetzen, ist nicht neu [12, 21]. Zeitweilige Unterdrückung kann ein Zeichen von Kompetenz und innerhalb einer fordernden Situation hilfreich sein, indem Kontrolle über die eigenen Gefühle bewahrt wird [4]. Dies erleichtert jedoch nicht zwangsläufig von der Erfahrung [8]. Emotionen zu unterdrücken heißt, sie später aufzuarbeiten.

\section{Bewältigungsstrategien}

Die einzige Bewältigungsstrategie, die Rettungskräfte gegenüber Studierenden stärker verwendeten, war Akzeptanz. Buruck und Dörfel heben Akzeptanz und Toleranz eigener Gefühle als wichtige Komponenten zur Bildung von Resilienz hervor [5]. Mitmansgruber und Kollegen argumentieren, dass Rettungskräfte aufgrund einer Nichtbewertung ihrer Emotionen eine akzeptierende Haltung innen gegenüber einnehmen [21]. Doch Akzeptanz betraf in unserer Studie nicht die Emotionen (z. B. 
Tab. 3 Korrelate von chronischem Stress

\begin{tabular}{|l|l|l|}
\hline Skala & $\begin{array}{l}\text { Rettungs- } \\
\text { kräfte } \\
(n=161)\end{array}$ & $\begin{array}{l}\text { Studie- } \\
\text { rende } \\
(n=56)\end{array}$ \\
\hline
\end{tabular}

Emotionale Reaktivität

\begin{tabular}{|l|l|l|}
\hline Ekelbilder & $0,23^{* *}$ & $-0,08$ \\
\hline Empathie & \multicolumn{3}{|l|}{} \\
\hline Mitgefühl & $0,34^{* *}$ & 0,18 \\
\hline Unbehagen & $0,51^{* *}$ & $0,50^{* *}$ \\
\end{tabular}

\begin{tabular}{|l|l|l|}
\hline Emotionsregulation & \multicolumn{1}{l|}{$0,51 * *$} & $0,50 * *$ \\
\hline Unterdrückung & $0,25^{* *}$ & 0,09 \\
\hline Bewäligungsstrategien
\end{tabular}

Bewältigungsstrategien

\begin{tabular}{|l|l|l|}
\hline Akzeptanz & 0,08 & $-0,30^{* *}$ \\
\hline Aktive Bewältigung & $-0,23^{* *}$ & $-0,31^{* *}$ \\
\hline Ablenkung & $0,21^{* *}$ & 0,07 \\
\hline Selbstbeschuldigung & $0,56^{* *}$ & $0,60^{* *}$ \\
\hline Verleugnung & $0,33^{* *}$ & 0,18 \\
\hline Alkohol \& Drogen & $0,33^{* *}$ & 0,21
\end{tabular}

Anmerkung. Bivariate Korrelation nach Pear-

son von 0 (kein Zusammenhang) bis \pm 1 (perfekter Zusammenhang)

${ }^{* *} p<0,01$

Akzeptanz des empfundenen Ekels vor einer Verletzung), sondern die Situationen (z. B. dass Konfrontation mit ekelerregenden Verletzungen zum Beruf gehört [17]). Dunn und Kollegen fanden in ihrer Studie mit Gemeindehelfern, dass Akzeptanz von kritischen Ereignissen eine Form von Nichtregulation darstellen könnte und negative Gefühle sowohl in einer Situation als auch danach aufrechterhält [8]. Möglicherweise akzeptieren dagegen Rettungskräfte solche Ereignisse vordergründig als festen Bestandteil ihres Berufs. Diese Art der Akzeptanz stellte sich als unabhängig vom Stresserleben heraus. Zudem sind entsprechend unseren Ergebnissen verschiedene maladaptive Bewältigungsstrategien mit stärkerem chronischem Stress assoziiert, in diesem Falle Ablenkung, Selbstbeschuldigung, Verleugnung und Konsum von Alkohol und Drogen $[9,16]$.

Kirby und Kollegen betonen, dass ein breites Repertoire an adaptiven Bewältigungsstrategien die Resilienz der Rettungskräfte fördert, etwa durch Problemlösen, Optimismus oder das Aufsuchen von Unterstützung [16]. Der Kern von Bewältigung liegt darin, solche Strategien dem Kontext entsprechend auszuwählen [2], und insbesondere die aktive Auseinandersetzung nicht nur mit dem Ereignis, sondern auch mit Emotionen ist eine beson- ders effektive, proaktive Vorgehensweise [5]. Auch in unserer Stichprobe wurden viele weitere adaptive Strategien wie das Aufsuchen emotionaler und instrumenteller Unterstützung berichtet. Es überraschte uns zunächst, dass diese meist weniger zum Einsatz kamen als bei Studierenden. Dies ließe sich möglicherweise auf eine geringere Beanspruchung aufgrund niedrigerer Reaktivität und Empathie zurückführen, sodass nachträgliche Bewältigung weniger dringlich ist. Zuletzt ist die aktive Bewältigung in Zusammenhang mit vermindertem Stress hervorzuheben. Durch den eigenen Beitrag (ggf. ergänzt durch fremde Unterstützung oder interne Beratungsdienste [11]) sei hier eine selbstwirksame Auseinandersetzung mit Situation und Emotion vorteilhaft [2, 3, 12, 14]. So können Rettungskräfte die eigenen emotionalen Kompetenzen fördern und ihre Ressourcen ausbauen, sodass ihre eigenen Emotionen und die der anderen den Beruf bereichern, statt zu belasten.

\section{Limitationen und Empfehlungen}

Bezüglich der Ausprägungen in Empathie, Emotionsregulation und Bewältigung sind keine Kausalschlüsse auf die Berufstätigkeit im Rettungsdienst möglich. So bleibt unklar, ob die gefundenen Resultate auf die Berufserfahrungen der Rettungskräfte zurückzuführen sind oder ob sie bereits in der Ausbildung bestehen [28]. Zudem schränken Verschiedenheiten beider Stichproben (z. B. Geschlecht und Alter) die Vergleichbarkeit ein. Empfohlen sei hier, in Zukunft auch andere Berufsgruppen zum Vergleich heranzuziehen, bspw. Personal mit unterschiedlich intensiver Emotionsarbeit und Patientenkontakt (z. B. Krankenpfleger, Anästhesisten, Feuerwehr). Dabei könnten auch kognitive Aspekte der Empathie (nüchterne Perspektivübernahme) und Kontrollempfinden durch emotionale Distanzierung untersucht werden [3]. Das verwendete Bildmaterial ist zwar validiert, jedoch kann es hinsichtlich mancher Inhalte (z. B. bedrohliche Tiere) und der Intensität wohl kaum mit der tatsächlichen Einsatzsituation verglichen werden. Die Reaktivität könnte mit Videomaterial repliziert und mit objektiven (z. B. Herzrate, Hautleitfähigkeit) oder qualitativen Maßen (z. B. Interviews) ergänzt werden.
Fazit für die Praxis

- Rettungskräfte berichteten ein mittleres chronisches Stresserleben, welches im Durchschnitt niedriger ausfiel als in der studentischen Vergleichsstichprobe.

- Rettungskräfte scheinen aufgrund einer geringeren (aber nicht grundsätzlich niedrigen) affektiven Empathie sowie geringerer Reaktivität auf ekelauslösendes Bildmaterial (menschliche Verletzungen) imstande, emotionale Belastungen von sich abzuschirmen.

- Durch weniger emotionales Involvement könnten Strategien zur Bewältigung von belastenden Situationen, die auch nach dem Einsatz anhalten, weniger dringlich sein.

- Die Akzeptanz schwieriger Situationen ist die einzige Bewältigungsstrategie, die von Rettungskräften stärker eingesetzt wurde als von der studentischen Vergleichsstichprobe. Für den Umgang mit chronischem Stress scheint allerdings nur die aktive Bewältigung eine protektive Rolle einzunehmen.

- Strategien zur aktiven Bewältigung könnten durch regelmäßige Beratung und kollegiale Unterstützung vermittelt werden.

Korrespondenzadresse

Juliane Völker

Fachbereich I - Psychologie, Universität Trier Universitätsring 15, 54296 Trier, Deutschland voelker@uni-trier.de

Funding. Open Access funding enabled and organized by Projekt DEAL.

\section{Einhaltung ethischer Richtlinien}

Interessenkonflikt. J. Völker und M.E.F. Flohr-Devaud geben an, dass kein Interessenkonflikt besteht.

Diese Studie wurde mit menschlichen Probanden unter Beachtung der ethischen Richtlinien der World Medical Association von 2013 („Declaration of Helsin$\mathrm{ki}^{\prime \prime}$ ) durchgeführt.

Open Access. Dieser Artikel wird unter der Creative Commons Namensnennung 4.0 International Lizenz veröffentlicht, welche die Nutzung, Vervielfältigung, Bearbeitung, Verbreitung und Wiedergabe in jeglichem Medium und Format erlaubt, sofern Sie den/die ursprünglichen Autor(en) und die Quelle ordnungsgemäß nennen, einen Link zur Creative Commons Lizenz beifügen und angeben, ob Änderungen vorgenommen wurden.

Die in diesem Artikel enthaltenen Bilder und sonstiges Drittmaterial unterliegen ebenfalls der genannten Creative Commons Lizenz, sofern sich aus der Abbildungslegende nichts anderes ergibt. Sofern das betreffende Material nicht unter der genannten Creative Commons Lizenz steht und die betreffende Handlung 
nicht nach gesetzlichen Vorschriften erlaubt ist, ist für die oben aufgeführten Weiterverwendungen des Materials die Einwilligung des jeweiligen Rechteinhabers einzuholen.

Weitere Details zur Lizenz entnehmen Sie bitte der Lizenzinformation auf http://creativecommons.org/ licenses/by/4.0/deed.de.

\section{Literatur}

1. Abler B, Kessler H (2009) Emotion Regulation Questionnaire - Eine deutschsprachige Fassung des ERQ von Gross und John. Diagnostica 55(3):144-152

2. AlexanderDA, KleinS (2001) Ambulance personnel and critical incidents. Br J Psychiatry 178(1):76-81

3. Avraham N, Goldblatt H, Yafe E (2014) Paramedics' experiences and coping strategies when encountering critical incidents. Qual Health Res 24(2):194-208

4. Beck T, Riedl D, Exenberger $S$ et al (2018) Führungskräfte im Rettungsdienst sind anders als „normale" Einsatzkräfte. Notfall Rettungsmed 21(4):298-302

5. Buruck G, Dörfel D (2018) Emotionsregulation in der Arbeit am Beispiel Rettungsdienst

6. Darius S, Balkaner B, Böckelmann I (2020) Psychische Beeinträchtigungen infolge erhöhter Belastungen bei Notärzten. Notfall Rettungsmed 24(2):143-149

7. Decety J, Yang C-Y, Cheng Y (2010) Physicians down-regulate their pain empathy response: an event-related brain potential study. Neuroimage 50(4):1676-1682

8. Dunn BD, Billotti D, Murphy V et al (2009) The consequences of effortful emotion regulation when processing distressing material: a comparison of suppression and acceptance. Behav Res Ther 47(9):761-773

9. Essex B, Scott LB (2008) Chronic stress and associated coping strategies among volunteer EMS personnel. Prehosp Emerg Care 12(1):69-75

10. Faul F, Erdfelder E, Lang A-G et al (2007) G*Power 3 : a flexible statistical power analysis program for the social, behavioral, and biomedical sciences. Behav Res Methods 39(2):175-191

11. Häller P, Michael T, Koechlin KB (2009) PTBS und komorbide Beschwerden bei Rettungssanitätern. Verhaltensther Verhaltensmed 30(4):403-417

12. Halpern J, Gurevich M, Schwartz B et al (2009) What makes an incident critical for ambulance workers? Emotional outcomes and implications for intervention. Work Stress 23(2):173-189

13. Hering T, Beerlage I, Kleiber D (2011) Arbeitsanforderungen und Ressourcen im Rettungsdienst. ZGesundheitspsychol 19(4):159-172

14. Jonsson A, Segesten K (2004) Guilt, shame and need for a container: a study of post-traumatic stress among ambulance personnel. Accid Emerg Nurs 12(4):215-223

15. Karutz H, Overhagen M, Stum J (2013) Psychische Belastungen im Wachalltag von Rettungsdienstmitarbeitern und Feuerwehrleuten. Präv Gesundheitsf 8(3):204-211

16. Kirby R, Shakespeare-Finch J, Palk G (2011) Adaptive and maladaptive coping strategies predict posttrauma outcomes in ambulance personnel. Traumatology 17(4):25-34

17. Knoll N, Rieckmann N, Schwarzer R (2005) Coping as a mediator between personality and stress

\section{Emotions in emergency medical services personnel. Emotional reactivity, empathy, and strategies compared to students}

Background: During emergency missions, rescue workers must often deal with emotionally stressful situations and their own reactions to human suffering. We examined rescue workers regarding their empathy and emotional reactivity, as well as strategies for emotion regulation and coping with difficult situations and correlated these with chronic experiences of stress. They were compared to a sample of students who had no medical experience.

Methods: Stress, empathy, emotion regulation and coping strategies were assessed using questionnaires. Emotional reactivity was investigated experimentally with standardized images that evoke disgust, sadness, fear, or joy, which were rated on a multilevel scale from pleasant to unpleasant.

Results: Rescue workers ( $n=161$ ) experienced less stress, were less empathetic, and used strategies for emotion regulation and coping less or similarly to students $(n=56)$. They also found disgusting and sad images less unpleasant. More reactivity to disgusting images, more empathy, emotion suppression, and avoidant coping strategies were associated with more stress.

Conclusion: A decreased, but not low empathy can be helpful for emotional shielding during an emergency mission, while afterwards an active examination of one's own emotions and the critical incidents appears protective against stress.

\section{Keywords}

Emergency rescue $\cdot$ Chronic stress $\cdot$ Emotion regulation · Coping strategies · Prevention

outcomes: a longitudinal study with cataract surgery patients. Eur J Pers 19(3):229-247

18. Kus L, Henderson L, Batt AM (2019) Empathy in paramedic practice: an overview. J Paramed Pract 11(4):1-5

19. Lang PJ, Bradley MM, Cuthbert BN (1997) International Affective Picture System (IAPS): technical manual and affective ratings

20. Mikels JA, Fredrickson BL, Larkin GR et al (2005) Emotional category data on images from the International Affective Picture System. Behav Res Methods 37(4):626-630

21. Mitmansgruber $H$, Beck TN, Schüßler G (2008) "Mindful helpers": Experiential avoidance, metaemotions, and emotion regulation in paramedics. JRes Pers 42(5):1358-1363

22. Paulus C (2009) Der Saarbrücker Persönlichkeitsfragebogen SPF (IRI) zur Messung von Empathie

23. Questback GmbH (2020) EFS survey. Questback $\mathrm{GmbH}, \mathrm{Köln}$

24. Regehr C, Goldberg G, Hughes J (2002) Exposure to human tragedy, empathy, and trauma in ambulance paramedics. Am J Orthopsychiatry 72(4):505-513

25. Reinhard F, Maercker A (2003) Sekundäre Traumatisierung, Posttraumatische Belastungsstörung, Burnout und Soziale Unterstützung bei medizinischem Rettungspersonal. Z Med Psychol $12: 1-8$

26. Roth K, Baier N, Felgner S et al (2020) Der Zusammenhang zwischen Sicherheitskultur und Burnout-Risiko: Eine Befragung nicht-ärztlicher Mitarbeiterim Rettungsdienst. Gesundheitswesen 82:1-9. https://doi.org/10.1055/a-1276-0817

27. Schulz P, SchlotzW, Becker P (2004) Trierer Inventar zum chronischen Stress. Hogrefe, Göttingen

28. Williams B, Lau R, Thornton E et al (2017) The relationship between empathy and burnout-Lessons for paramedics: a scoping review. Psychol Res Behav Manag 10:329-337
29. World Medical Association (2013) World Medical Association Declaration of Helsinki: Ethical principles for medical research involving human subjects. JAMA 310(20):2191-2194 
Hier steht eine Anzeige.

黑 Springer 